\title{
EXTRACCIÓN QUIRÚRGICA \\ DE LA NEOVASCULARIZACIÓN COROIDEA PERIPAPILAR EN PACIENTES JÓVENES
}

\section{SURGICAL REMOVAL OF PERIPAPILLARY CHOROIDAL NEOVASCULARIZATION IN YOUNG PATIENTS}

\author{
RUIZ-MORENO JM ${ }^{1,3}$, AMAT-PERAL P ${ }^{2}$, LUGO FL ${ }^{2}$, MONTERO JA ${ }^{3}$
}

\begin{abstract}
RESUMEN
Propósito: Analizar el resultado anatómico y visual después de la cirugía subretiniana de la neovascularización coroidea (NVC) peripapilar no asociada a Degeneración Macular Asociada a la Edad (DMAE). Métodos: Se estudiaron retrospectivamente cinco ojos de cinco pacientes con NVC (neovascularización coroidea) tratados mediante cirugía subretiniana.

Resultados: La media de edad de los pacientes fue de 32 años (rango de 11 a 49, DE 14,5 años) y la media del seguimiento fue de 31,4 meses (rango de 8 a 48 , DE 20,8 meses). La Mejor agudeza visual corregida antes de la cirugía fue de 0,05 (rango de 0,001 a 0,16, DE 0,07 ) y de 0,64 (rango de 0,2 a 0,9 , DE 0,26$)$ después de la cirugía ( $\mathrm{p}=0,005$; $\mathrm{t}$ de Student para datos pareados). No hubo incidencias intraoperatorias ni postoperatorias.

Conclusiones: La cirugía de extracción de membranas debe ser considerada entre las opciones terapéuticas para tratar la NVC peripapilar no relacionada con DMAE.
\end{abstract}

Palabras clave: Neovascularización coroidea peripapilar, vitrectomía, cirugía subretiniana, neovascularización coroidea no asociada a DMAE.

\begin{abstract}
Purpose: To analyze anatomical and visual outcomes after subretinal surgery for peripapillary choroidal neovascularization $(\mathrm{CNV})$ not associated with age-related macular degeneration (AMD).

Methods: Five eyes from five patients with peripapillary CNV who had been treated by subretinal surgery were retrospectively analyzed.

Results: The patients averaged 32 years of age (range, 11 to 49 , SD 14.5 years) and mean followup was 31.4 months (range, 8 to 48 , SD 20.8 months). Best corrected visual acuity was 0.05 (range, 0.001 to 0.16 , SD 0.07 ) before surgery and 0.64 (range, 0.2 to $0.9, \mathrm{SD} 0.26)$ after surgery $(\mathrm{p}=0.005$; Student's t test for paired data). Neither intraoperative nor postoperative incidences occurred.

Conclusions: Surgical removal may be considered one of the therapeutic options to treat peripapillary CNV not related to AMD (Arch Soc Esp Oftalmol 2009; 84: 39-42).
\end{abstract}

Key words: Peripapillary choroidal neovascularization, vitrectomy, subretinal surgery, non-AMDrelated choroidal neovascularization.

\footnotetext{
Recibido: 14/9/07. Aceptado: 22/12/08.

Instituto Oftalmológico de Alicante, VISSUM, Unidad de Vítreo-Retina. Alicante

1 Catedrático de Oftalmología. Universidad de Castilla-La Mancha (UCLM).

${ }^{2}$ Licenciado en Medicina.

3 Doctor en Medicina.

Correspondencia:

Pedro Amat-Peral

Calderón de la Barca, 29, 2. ${ }^{\circ} \mathrm{A}$

03004 Alicante

España

E-mail: pamatp@yahoo.es
} 


\section{INTRODUCCIÓN}

La neovascularización coroidea (NVC) adyacente a la papila es menos frecuente que la NVC submacular y está comunmente asociada a la Degeneración Macular Asociada a la Edad (DMAE). La NVC peripapilar en pacientes jóvenes está usualmente asociada a otras patologías oculares como son la miopía patológica, el síndrome de presunta histoplasmosis ocular (SPHO), drusas del disco óptico, estrías angioides y edema papilar (1).

Se han descrito diferentes alternativas terapéuticas para el tratamiento de la NVC peripapilar de cualquier etiología, incluyendo fotocoagulación con láser térmico (1), terapia fotodinámica (TFD) (2), cirugía subretiniana (2-4) y recientemente fármacos antiangiogénicos.

Nosotros presentamos los resultados de una serie consecutiva de NVC peripapilar no asociada con DMAE en pacientes jóvenes, menores de 50 años de edad después de la extracción quirúrgica de la NVC.

\section{SUJETOS, MATERIAL Y MÉTODOS}

Se revisaron de manera retrospectiva las historias clínicas, las tomografías de coherencia óptica (OCT), las angiografías fluoresceínicas (AGF) y las retinografías de cinco pacientes jóvenes (cinco ojos) con NVC peripapilar tratada con cirugía subretiniana, tras obtener el consentimiento informado por escrito para poder realizar el estudio.

El consentimiento informado por escrito se obtuvo después de explicar la naturaleza del procedimiento antes de la cirugía. Los procedimientos se realizaron de acuerdo con los estándares éticos de la Declaración de Helsinki de 1975, revisada en 1983. Bajo anestesia peribulbar (un caso bajo anestesia general) se practicaba una vitrectomía vía pars plana con desprendimiento de la hialoides posterior y tras ello una retinotomía pequeña por la que, con unas pinzas subretinianas se despegaba y extraía la NVC. Finalmente se realizaba un intercambio de fluido-aire y se posicionaba a los pacientes durante 72 horas.

\section{RESULTADOS}

La media de edad de los pacientes fue de 32 años (rango de 11 a 49, DE 14,5 años). Tres eran mujeres y dos hombres (tabla I). La media del seguimiento fue de 31,4 meses (rango de 8 a 48, DE 20,8 meses). Previo a la cirugía, la mácula estaba afectada por hemorragia en un caso y por exudación en dos casos. La etiología de la NVC fue desconocida en tres casos; un caso se asoció a drusas del disco óptico y un caso a edema papilar (tabla I).

La mejor agudeza visual corregida (MAVC) antes de la cirugía fue de 0,05 (rango de 0,001 a 0,16 , DE 0,07 ) y de 0,64 (rango de 0,2 a 0,9 , DE $0,26)$ al final del periodo de seguimiento $(p=0,005$; $\mathrm{t}$ de Student para datos pareados).

Una mujer de 38 años (caso \#2) presentaba un escotoma central en su ojo derecho de quince días de evolución. La MAVC era de movimiento de manos y el examen del fondo de ojo mostraba un edema papilar y una hemorragia subretiniana próxima al disco óptico de su ojo derecho (Figura 1A). La AGF revelaba una NVC peripapilar que afectaba a la mácula (fig. 1B y C) con incremento del espesor retiniano en la OCT (fig. 1D). La NVC se extrajo quirúrgicamente como se ha descrito. Dos meses después la MAVC era de 0,65 (fig. 1E) con un espesor macular normal (fig. 1F).

Tabla 1

\begin{tabular}{|c|c|c|c|c|c|}
\hline Paciente \# & 1 & 2 & 3 & 4 & 5 \\
\hline Edad (años) & 11 & 38 & 25 & 49 & 37 \\
\hline Sexo & Hombre & Mujer & Mujer & Hombre & Mujer \\
\hline MAVC inicial (Decimal) & Cuenta dedos & Movimiento de manos & 0,1 & Cuenta dedos. & 0,16 \\
\hline Etiología & Drusas & Edema papila & No & No & No \\
\hline Macula & Afectada & Afectada & Afectada & Normal & Normal \\
\hline Fecha cirugía & Junio '04 & Diciembre '05 & Enero '98 & Enero '00 & Diciembre' 00 \\
\hline MAVC 2 meses (Decimal) & 0,4 & 0,65 & 0,3 & 0,16 & 0,5 \\
\hline MAVC final (Decimal) & 0,7 & 0,7 & 0,9 & 0,2 & 0,7 \\
\hline Seguimiento (meses) & 29 & 8 & 37 & 35 & 48 \\
\hline Tratamiento previo & TFD (2 veces) & No & No & No & No \\
\hline
\end{tabular}

MAVC: Mejor agudeza visual corregida. TFD: Terapia fotodinámica. 


\section{DISCUSIÓN}

La NVC peripapilar representa aproximadamente un $10 \%$ de todos los casos de NVC (3). Esta patología puede resolverse espontáneamente o crecer e invadir la mácula, causando hemorragia submacular o desprendimiento seroso macular reduciendo la agudeza visual.

La TFD se ha usado profusamente para tratar la NVC submacular; sin embargo, la TFD no es considerada apropiada para tratar la NVC si está dentro de las $200 \mu \mathrm{m}$ desde el disco óptico porque hay un riesgo mayor de dañar las fibras del mismo (4). Un paciente de nuestra serie había recibido dos sesiones de TFD previamente para una NVC extrafoveolar localizada a más de $200 \mu \mathrm{m}$ del disco óptico; la NVC alcanzó la papila después de la segunda sesión de TFD y por ello decidimos extraerla quirúrgicamente.

Kokame ha demostrado que la función foveal se puede preservar si la NVC se extrae antes de que la fóvea se afecte (5). El mayor inconveniente de la cirugía subretiniana en la NVC subfoveal es el trauma quirúrgico al epitelio pigmentario de la retina (EPR). La cirugía subretiniana para tratar la NVC peripapilar puede conseguir la extracción completa de la NVC sin afectar al EPR subfoveal ni a la coriocapilar, preservando la agudeza visual. En nuestra pequeña serie la mácula estaba afectada en tres casos por fluido subretiniano y hemorragia, las cuales desaparecieron después de la cirugía de la NVC. En el caso 2, descrito previamente, se observa mejoría de la agudeza visual al retirar la NVC peripapilar que no alcanzaba la fóvea y la hemorragia adyacente subfoveal.

Kies y Bird han demostrado recidiva de la NVC en el $50 \%$ de los ojos a los que se le realizó fotocoagulación con éxito (6). Unas de las ventajas de la cirugía subretiniana es que la NVC puede ser extraída completamente disminuyendo el riesgo de recurrencias tempranas.

La cirugía subretiniana ha demostrado ser eficaz en el manejo de la NVC peripapilar en aquellos casos en los que la fotocoagulación con láser térmico o la TFD no se aconsejan o han fallado. Bains analizó diecisiete ojos de pacientes mayores después de la extracción quirúrgica de la NVC en los que se observó una estabilización o mejoría de la MAVC en más de un tercio de los casos (7). Atebara demostró en un estudio retrospectivo de diecisiete ojos con NVC peripapilar asociada a SPHO en pacientes jóvenes a los que se les había realizado cirugía de extracción de
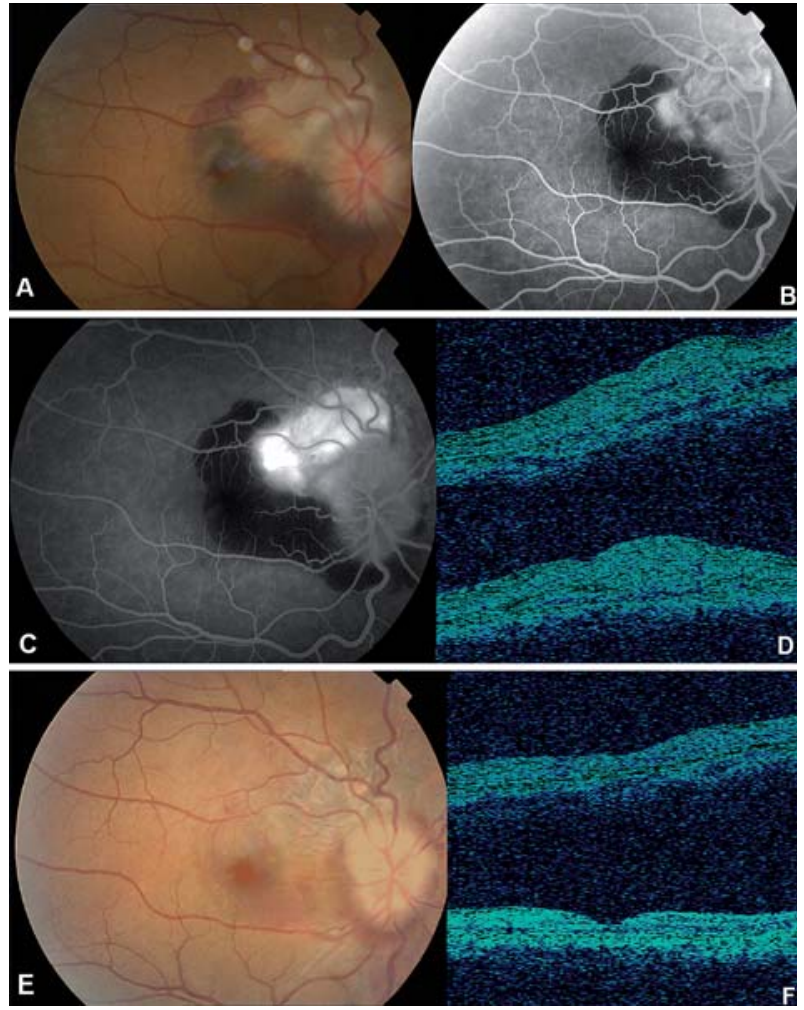

Fig. 1: A: Fotografía en color del fondo de ojo del paciente \#2 mostrando la neovascularización coroidea $(N V C)$ con hemorragia subretiniana. B-C: Angiografía fluoresceínica (AGF) (tiempos medios y tardios) mostrando fuga de contraste desde la NVC. D: Tomografía de coherencia óptica (OCT) vertical (arriba) y horizontal (abajo) mostrando el espesor retiniano. E: Fotografía en color del fondo de ojo del paciente dos meses después de la extracción de la NVC. F: OCT vertical (arriba) y horizontal (abajo) después de la cirugía mostrando un espesor retiniano normal.

la membrana neovascular (8). En esta serie, la MAVC fue de 20/40 o mejor en el 50\% de los ojos con extensión subfoveal de la NVC, y 20/20 en tres ojos con NVC extrafoveal. No obstante, Essex (9) et al. observando los resultados en un estudio de seguimiento a dos años tras extracción quirúrgica de NVC no relacionada a DMAE, concluyeron que aparecían recurrencias hasta en el 33\% de los casos.

La llegada de los agentes antiangiogénicos, como son el pegaptanib, ranibizumab y bevacizumab puede mostrar una nueva alternativa al tratamiento de la NVC peripapilar. Sin embargo la cirugía subretiniana se demuestra como una terapia efectiva con buenos resultados anatómicos y visuales y una reducción de la tasa de recurrencia. Hemos encontrado 
una mejoría de la MAVC en todos los casos de nuestra serie, sin efectos adversos durante el seguimiento. La cirugía subretiniana debe ser considerada una de las opciones terapeúticas para tratar NVC no asociada a DMAE. Sin embargo, hay que tener en cuenta los potenciales riesgos, como son el desprendimiento de retina, los agujeros retinianos y el edema macular quístico.

Considerando que nuestra serie, como otras series presentadas, es corta, serían necesarios estudios randomizados, multicéntricos, con mayor tiempo de seguimiento y mayor número de pacientes para demostrar la eficacia real de esta terapia, vs otras posibilidades como son la fotocoagulación con láser térmico, la TFD o el anti-factor de crecimiento vascular endotelial.

\section{BIBLIOGRAFÍA}

1. Bhatt NS, Al-Shirawi NM, Diamond JG. Krypton laser photocoagulation of peripapillary choroidal neovascular membrane. Ophthalmic Surg Lasers 1999; 30: 56-58.
2. Rosenblatt BJ, Shah GK, Blinder K. Photodynamic therapy with verteporfin for peripapillary choroidal neovascularization. Retina 2005; 25: 33-37.

3. Kertes PJ. Massive peripapillary subretinal neovascularization: an indication for submacular surgery. Retina 2004; 24: 219-225.

4. Photodynamic therapy with verteporfin for age-related macular degeneration. American Academy of Ophthalmology. Ophthalmology 2000; 107: 2314-2317.

5. Kokame GT,Yamaoka S. Subretinal surgery for peripapillary subretinal neovascular membranes. Retina 2005; 25: 564-569.

6. Kies JC, Bird AC. Juxtapapillary choroidal neovascularization in older patients. Am J Ophthalmol 1988; 105: 1119 .

7. Bains HS, Patel MR, Singh H, Marcus DM. Surgical treatment of extensive peripapillary choroidal neovascularization in elderly patients. Retina 2003; 23: 469-474.

8. Atebara NH, Thomas MA, Holekamp NM, Mandell BA, Del Priore LV. Surgical removal of extensive peripapillary choroidal neovascularization associated with presumed ocular histoplasmosis syndrome. Ophthalmology 1998; 105: 1598-1605.

9. Essex RW, Tufail A, Bunce C, Aviward GW. Two-year results of surgical removal of choroidal neovascular membranes related to non-age-related macular degeneration. Br J Ophthalmol 2007; 91: 649-654. 